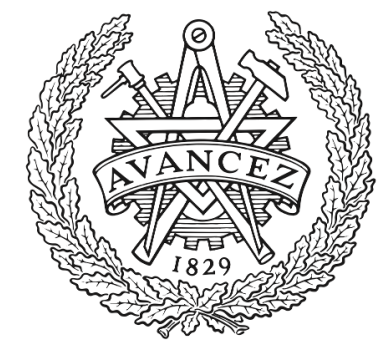

CHALMERS

\title{
Direct-Coupled Cavity Filter in Ridge Gap Waveguide
}

Downloaded from: https://research.chalmers.se, 2023-04-26 11:49 UTC

Citation for the original published paper (version of record):

Sorkherizi, M., Khaleghi, A., Kildal, P. (2014). Direct-Coupled Cavity Filter in Ridge Gap

Waveguide. IEEE Transactions on Components, Packaging and Manufacturing Technology, 4(3): 490-495. http://dx.doi.org/10.1109/tcpmt.2013.2284559

N.B. When citing this work, cite the original published paper.

(O2014 IEEE. Personal use of this material is permitted.

However, permission to reprint/republish this material for advertising or promotional purposes 


\title{
Direct-Coupled Cavity Filter in Ridge Gap Waveguide
}

\author{
Milad Sharifi Sorkherizi, Ali Khaleghi, Member, IEEE, and Per-Simon Kildal, Fellow, IEEE
}

\begin{abstract}
This paper describes a novel design for a directcoupled cavity filter realization using ridge gap waveguide technology. A ridge gap waveguide transmission line with two coaxial feed connectors is designed and operated within the frequency band of 10-13 GHz. A cavity is coupled to the transmission line in order to achieve bandstop filter characteristic. Thereafter, the structure of the filter is modified in a way to generate a bandpass feature. The final manufactured prototype is a forth order bandpass filter, operating at the center frequency of $11.59 \mathrm{GHz}$ with a bandwidth of $72 \mathrm{MHz}$. The proposed design has potential applications in channeling filters for telecommunication satellites.
\end{abstract}

Index Terms_ridge gap waveguide, direct-coupled cavity filter, bandpass filter.

\section{INTRODUCTION}

$\mathrm{R}$ ecently a new metamaterial-based guiding structure in gap between two parallel metal plates has been introduced [1], [2]. The ridge gap waveguide supports quasiTEM wave propagation in the air gap between the parallel plates. The lower plate is textured with a 2-D periodic structures as well as intermediate ridges. The 2-D periodic structures introduce artificial magnetic conductor (AMC) boundary conditions that together with the smooth upper plate produce a stopband for parallel-plate modes and confine the energy along the guiding ridges. The proposed waveguiding structure can be realized without any dielectric material and no electrical connection between the plates is required [2]. These features highlight this technology as a suitable candidate for millimeter and submillimeter band guiding structure for realizing microwave components. Another interesting feature of ridge gap technology is its ability for integration and packaging as it can be designed to be completely enclosed.

The periodic texture in the lower plate of the ridge gap waveguide introduces when integrated with the upper metal plate, a stopband in which no guiding and cavity mode between the two parallel plates can be excited. Different configurations can be used to achieve the desired stopband

Manuscript received October 9, 2001. M. Sharifi Sorkherizi and A. Khaleghi are with the Department of Electrical and Computer Engineering, K. N. Toosi University of Technology, Tehran, Iran (e-mail: milad.sharifi@hotmail.com, khaleghi@eetd.kntu.ac.ir).

P-.S Kildal is with the Department of Signals and Systems, Chalmers University of Technology, Gothenburg, Sweden (email: persimon.kildal@chalmers.se) characteristics, which design procedures and stopband calculations are presented in [3]. One desirable geometry of this periodic pattern is the so-called "bed of nails" which also has applications in microwave circuit packaging technology [4], [5].

One vital and challenging microwave component in a complicated communication system, is diplexer or multiplexer which serves as channel separator. The multiplexer simply can be defined as relatively narrow-band filters in a filter bank which are governing the characteristics of the communication transponder [6]. Hence, a significant effort is generally made to optimize their characteristics. To design a multiplexer, the filter channels usually are designed separately to achieve an acceptable performance. Then, they are interconnected to form a multiplexer configuration. The next step is to re-optimize the design or introduce the matching elements to achieve acceptable responses [7]. In this paper we concentrate on the first step that is the design of a single narrow band filter for a conventional channel in satellite communication.

The design is based on direct-coupled cavity filter geometry which characteristics have been widely studied in literature for waveguide cavities [8]-[10]. The main goal of our investigation is to achieve a fully integrated design based on ridge gap waveguide. There have been previous publications on gap waveguide filters, such as the microstrip gap waveguide packaged filter in [5] and the groove gap waveguide filter in [11]. The gap waveguides are in particular suitable for packaging of whole microwave modules with TX \& $\mathrm{Rx}$ amplifiers, such as demonstrated in [12]. Then, gap waveguide filters can readily be integrated in such packaging modules. The present direct-coupled cavity is also a kind of groove gap waveguide filter but it has a simpler mechanical design with fewer and thicker pins that are less expensive to manufacture for high frequency. In particular, we use here only one row of pins between each cavity resonator. Also the design is a waveguide based filter which is suitable for multiplexer configuration.

The paper is organized as follows. In section II we proposed a novel cavity structure which can easily be integrated with the ridge gap waveguide. The resonator is realized by introducing a defect in the surrounding periodic pattern around the guiding ridge. Different modes of this cavity are studied and the affecting factor in its resonance frequency is analyzed. The adjacent cavity to the ridge introduces a bandstop filter. In section III, another guiding ridge that can be coupled to the cavity is designed in order to 

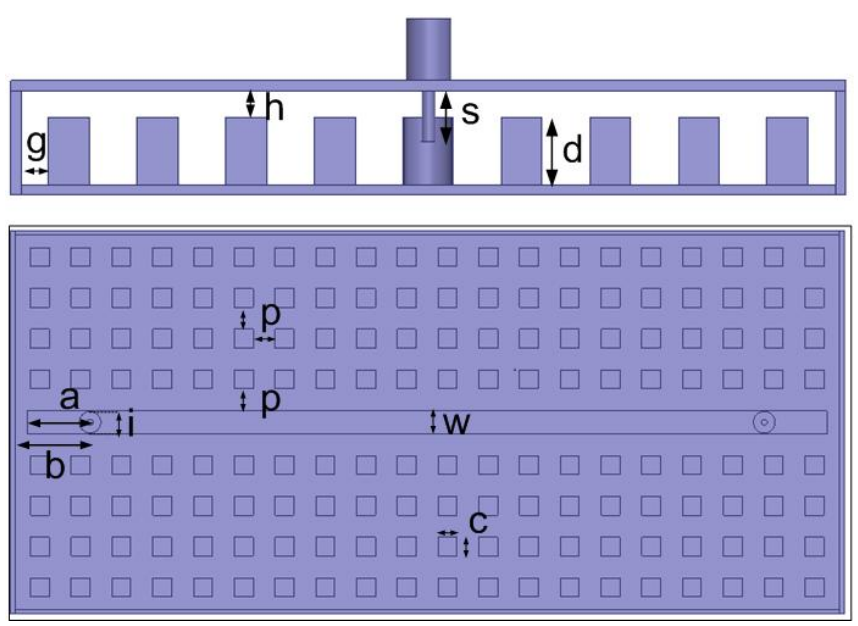

Fig. 1. Geometry of designed ridge gap waveguide for $10-13 \mathrm{GHz}$ band. a)Side view b)Top view. The parameters are defined as follow: $a=13 \mathrm{~mm}$, $\mathrm{b}=15.6 \mathrm{~mm}, \mathrm{c}=3.9 \mathrm{~mm}, \mathrm{~d}=6.5 \mathrm{~mm}, \mathrm{~g}=2.6 \mathrm{~mm}, \mathrm{~h}=1.3 \mathrm{~mm}, \mathrm{i}=4.48 \mathrm{~mm}, \mathrm{p}=4.55$ $\mathrm{mm}, \mathrm{s}=4.87 \mathrm{~mm}, \mathrm{w}=4.75 \mathrm{~mm}$.

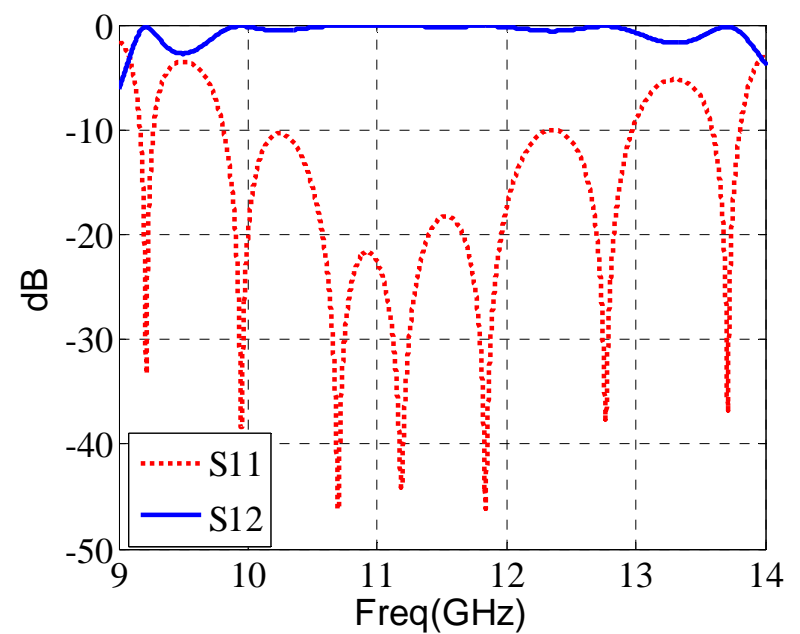

Fig. 2. Simulated S-parameters of the designed ridge gap waveguide (see Fig. 1).

achieve a bandpass filter. In section IV, multiple cavities with proper dimensions and couplings are adapted in series to yield higher order filter with a desired bandwidth. In section $\mathrm{V}$ the experimental results for an optimized forth order filter at the center frequency of $11.59 \mathrm{GHz}$ with a bandwidth of $72 \mathrm{MHz}$ are presented.

\section{CAVity Structure in Ridge GAP WAVEguide}

\section{A. Ridge Gap Waveguide in 10-13 GHz Frequency Band}

The design procedure of a ridge gap waveguide is introduced in [2]. Here, a new geometry is designed for operating in x-band, more specifically around the center frequency of $11.59 \mathrm{GHz}$, in order to demonstrate the concept of coupled cavity filter on a conventional channel in satellite communications. The dimensions of the $\mathrm{x}$-band prototype are shown in Fig.1. The bed of nails structure used in the waveguide can provide bandgap between 7.16-7.13 GHz.

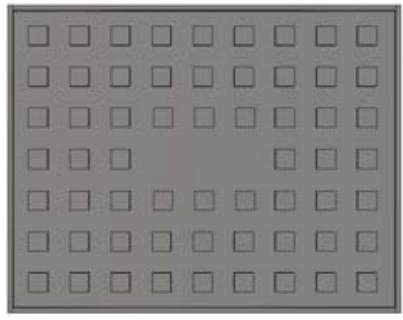

(a)

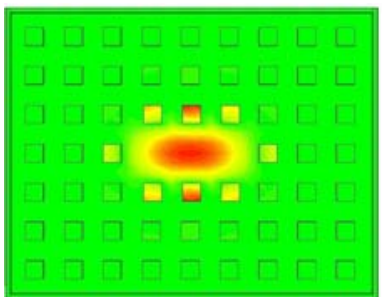

(b)

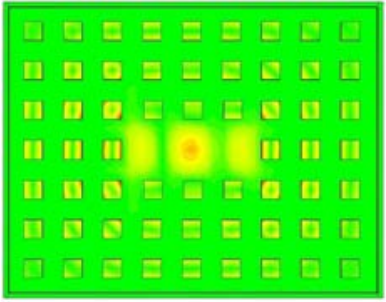

(d)

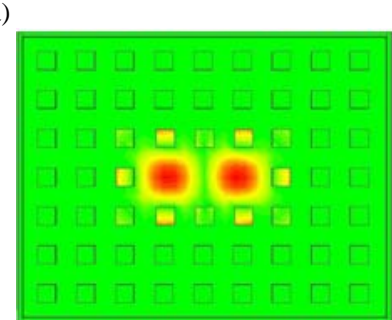

(c)

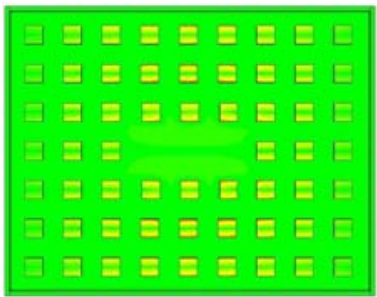

(e)
Fig. 3. (a): Proposed cavity which is realized by creating three defects in the finite periodic EBG structure of Ridge Gap waveguide. The upper lid is not shown. (b): Electrical filed distribution of the first mode. (c): Second mode. (d): Third mode. (e): Fourth mode.

TABLE I

FIRST FOUR MODES’ RESONANT FREQUENCIES AND Q-FACTORS OF THE CAVITY STRUCTURE PRESENTED IN FIG. 3

\begin{tabular}{|c|c|c|}
\hline Mode & Frequency( GHz) & Q-factor \\
\hline 1 & 12.3142 & $5.4561 \times 10^{3}$ \\
\hline 2 & 14.5637 & $6.0015 \times 10^{3}$ \\
\hline 3 & 17.1663 & $4.0539 \times 10^{3}$ \\
\hline 4 & 17.1775 & $3.9102 \times 10^{3}$ \\
\hline
\end{tabular}

However the operating frequency is limited by the coaxial feed excitation which is designed with respect to [13]. The Sparameters of the full wave simulated design is shown in Fig. 2. As shown the return loss is less than $10 \mathrm{~dB}$ for $10-13 \mathrm{GHz}$. In this frequency range the energy is confined in the air gap between the upper lid and the ridge.

\section{B. Proposed Cavity in Ridge Gap Waveguide}

Direct-coupled cavity filter is a well-known structure to demonstrate narrow-band filters [8]-[10]. The first step of filter design is to realize a cavity with the intended resonance frequency that can be coupled to the guided energy of waveguide and realized easily in mechanical matter.

At first glance the ridge gap structure shows a unique similarity to crystal photonics which were studied widely in recent years [14]. By this analogy, it seems that the realization of cavities in ridge gap structure can be done by creating a defect in the periodic structure as in crystal photonics [15]. To 
understand the situation, a defect is created in the periodic high-impedance surface of the ridge gap waveguide. Fig. 3-a shows this structure, which is a finite periodic surface in which three pins in the center is removed. The dimensions and period of the pins are as shown in Fig. 1. CST Eigenmode solver is used for calculations. The resonance frequencies of the first four modes above $9 \mathrm{GHz}$ and relating Q-factors are presented in Table I. The electric field distributions of the first four resonances are illustrated in Fig.3-b-e. As shown, the electric field is confined within the cavity for the first two resonance modes. For the upper modes, the created cavity is not responsible for the resonance as the frequency is out of the stopband for parallel-plate modes. In these modes the electric field is distributed within the metallic pins. Q-factor of the proposed cavity is high enough to satisfy the commercial specification of the radio links in x-band. The high $Q$ properties of gap waveguides are studied more thoroughly in [16] and [17].

A key factor for a cavity is the capability of resonance frequency tuning. For this purpose, the volume of the cavity is altered by keeping constant dimensions of the periodic texture. In this work we also keep the length and height of the cavity constant, and we adjust the resonance frequency by changing only the width (w) as shown in Fig. 4-a. We see that the width of the rods surrounding the cavity has been changed, and thereby the total width of the cavity can be controlled. Fig. 4-b shows the variation of the resonance frequency of the first mode versus $w(\mathrm{~mm})$. As shown, the resonance frequency reduces by increasing the cavity width.

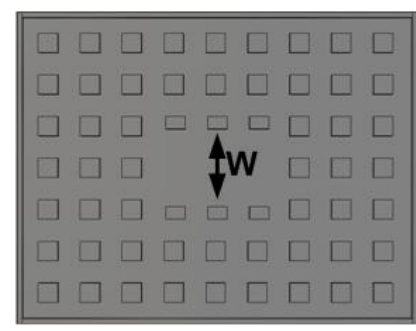

(a)

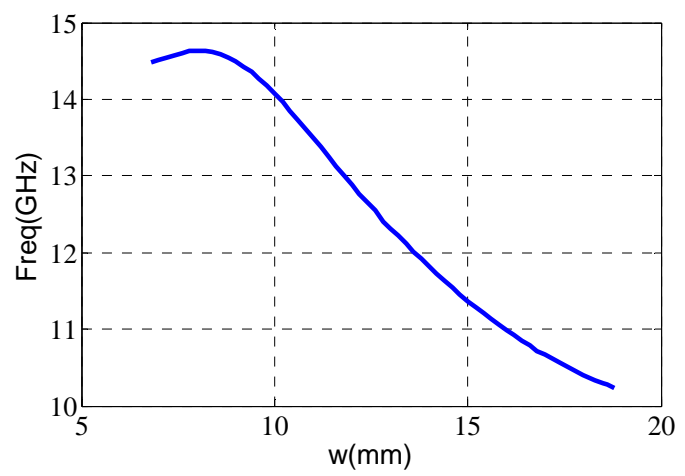

(b)

Fig. 4. (a): The cavity structure of Fig. 3 with variable width. The sizes of the surrounding pins control the size of the cavity. Other dimensions are identical to the presented parameters of the EBG surface in Fig. 1. (b): Resonant frequency of the first mode is plotted as a function of the width of the cavity.

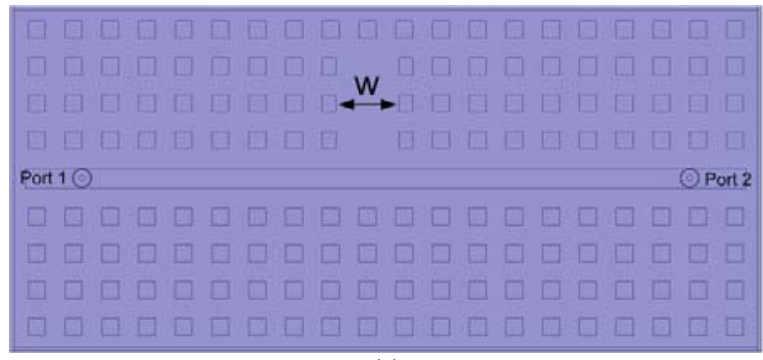

(a)

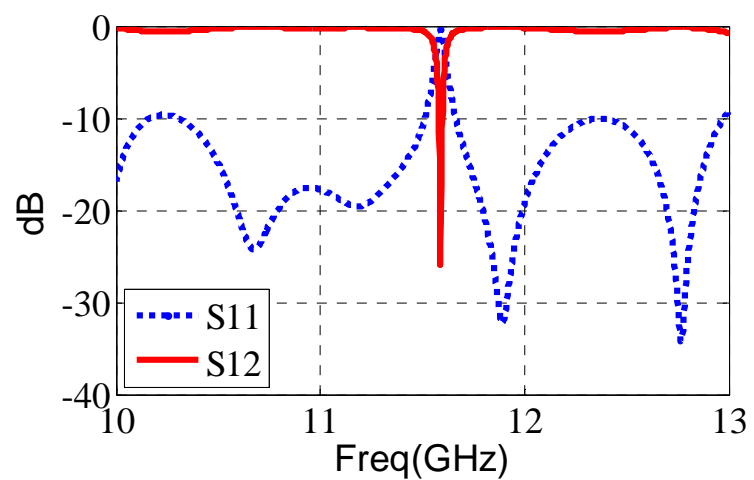

(b)

Fig. 5. (a): Bandstop filter by placing the designed cavity adjacent to the guiding ridge. (b): Simulated S-parameters which shows band elimination in $15.59 \mathrm{GHz}$. "w" is set to $14.58 \mathrm{~mm}$.

\section{Bandstop Filter Using the Designed Cavity}

Generally in rectangular waveguide structures the coupling from waveguide to cavity is achieved by a T-junction, which needs proper design itself. In ridge gap structure this can be acquired by simply positioning the defect near the ridge. Fig. 5 shows this geometry and the simulated transmission parameters using HFSS for the cavity length of $w=14.58 \mathrm{~mm}$. Fig. 4 anticipated the resonant frequency of $11.54 \mathrm{GHz}$ but as the geometry is perturbed by the existence of the ridge, the resonance occurs at $11.59 \mathrm{GHz}$. The coupled cavity geometry works as a band-eliminating filter which resonance frequency can be controlled by the cavity width $(w)$.

\section{Coupled CAVIty Filter ON Ridge Gap WAVEguide (BAND PASS FILTER)}

The next step is realizing a bandpass filter using the coupled cavity geometry. For this purpose, we introduce a new ridge to extract the trapped energy in the resonance frequency of the aforementioned cavity. The geometry of Fig. 6 is a solution that is identical to the geometry of Fig. 5, but another ridge is inserted in the vicinity of the cavity. The dimensions of the new ridge are identical to the main ridge. Also, to fix the resonance at $11.59 \mathrm{GHz}$ a little adjustment is needed in the width of the cavity, or in the length by altering the length of the second ridge. In Fig. 6-a the width (w) and length (l) of the cavity are equal to $14.82 \mathrm{~mm}$ and $23.80 \mathrm{~mm}$, respectively. The transmission results of this structure are plotted in Fig. 6. This is a poor result as the $S_{12}$ has at least 3 $\mathrm{dB}$ loss. By canceling the coaxial port at the end of the main ridge (specified by Port 3 in Fig.6) or by creating a short 


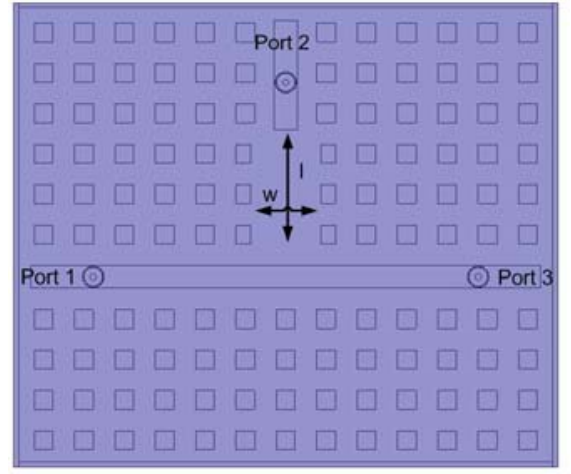

(a)

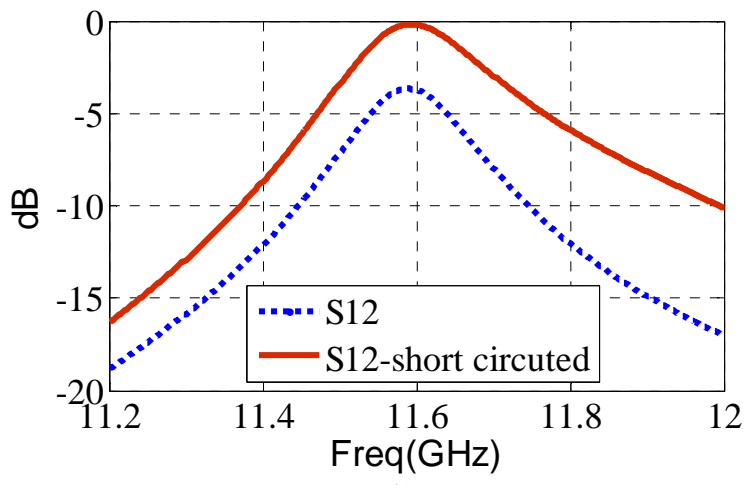

(b)

Fig. 6. (a): First order bandpass filter by placing another ridge near the cavity (b): Simulated $\mathrm{S}_{12}$ of the (a) and the short circuited prototype which is identical to (a) but the coaxial transition in port 3 is canceled.

circuit, the $S_{12}$ shows a significant improvement which is plotted in fig. 6-b. In this realization the important issue is the distance between the center of the cavity and the short circuit end. This distance should be adjusted to $(\mathrm{N}+0.5) \lambda_{\mathrm{g}}$ where $\lambda_{\mathrm{g}}$ is the effective wavelength in the parallel plate waveguide and $\mathrm{N}$ is an integer number. As the ridge gap waveguide supports quasi-TEM waves, the wavelength can be approximated by the wavelength in free space. This does not oblige very severe condition because the coupling area between the first guiding ridge and the cavity is large enough to tolerate few fraction of mismatch.

\section{Coupled CAVity Filter on Ridge Gap WAVEguide (FORTH ORDER BAND PASS FILTER)}

In order to achieve a practical narrow band filter one needs to employ extra cavities to introduce multiple poles to the filter. In Fig. 7 four identical cavities are aligned in cascade in order to realize a fourth order bandpass filter. The coupling between the cavities is controlled by the height of the rods that are placed between the cavities. Other dimensions of the rods are identical to other rods of the aforementioned pattern. This fact was used in the design to place the poles in the desired position in order to achieve a maximally flat response with center frequency of $11.59 \mathrm{GHz}$ and $72 \mathrm{MHz}$ bandwidth.

The simulations showed that two rows of rods in every side of each ridge are sufficient to create satisfactory artificial magnetic conductor (AMC) boundary conditions. Hence, in Fig. 7-a the total size of the design is reduced. The parameters of this design are presented in the caption for Fig. 7. The lengths of the cavities are identical in pairs, which means the first and the last cavities have same lengths so the two cavities in middle. The widths of the cavities are all identical and equal to " $w$ ". " $h$ " shows the height of the second coupling pin. Dimensions of other coupling pins are all identical to the pins of the periodic pattern surface (Fig.1). The exact parameter dimensions are presented in Fig. 7.

Different channels can be implemented in the proposed structure to introduce a channel multiplexer. The authors are working on implementing a complete channel multiplexer on this structure.

\section{EXPERIMENTAL RESULTS}

The designed model in Fig. 7-a was fabricated using CNC etching machine on Aluminum. Fig. 8 shows the physical realization of the filter that is comprised of two parts. The sidewalls are connected to the upper lid and are provided with several holes for connecting screws. The screws are only for mechanical enforcement and as mentioned in the introduction no electrical connection is needed between the plates. Two SMA connectors are inserted from the upper plate and the inners are placed inside the defined holes in the ridges as in Fig. 1.

The S-parameters were measured using a vector network analyzer. The experimental results along with simulations are

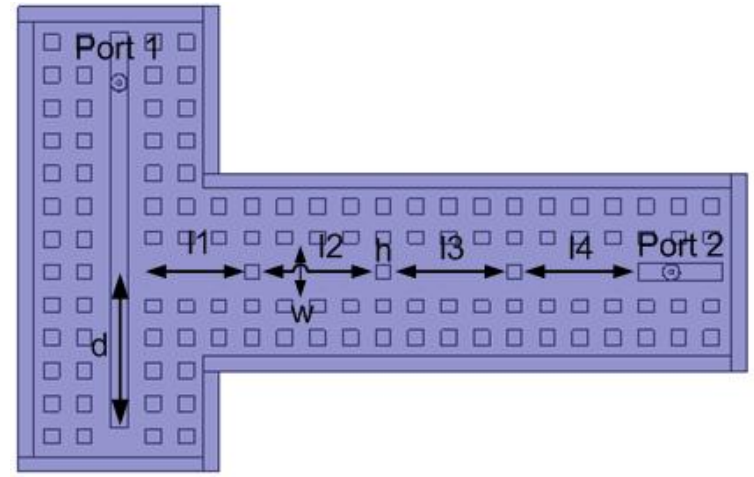

(a)

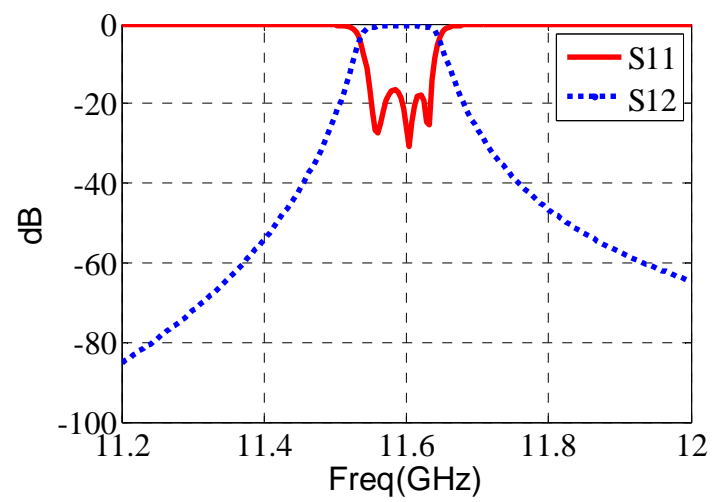

(b)

Fig. 7. (a): A fourth degree direct coupled cavity filter on ridge rap waveguide. The center frequency in $11.59 \mathrm{GHz}$ and the usable bandwidth (insertion loss $<0.7 \mathrm{~dB}$ ) is $72 \mathrm{MHz}$. The parameters are as follows: $\mathrm{l} 1=\mathrm{l} 4=29.98 \mathrm{~mm}, \mathrm{l}=\mathrm{l}=29.8 \mathrm{~mm}, \mathrm{w}=14.55 \mathrm{~mm}, \mathrm{~h}=6.9 \mathrm{~mm}, \mathrm{~d}=39.85 \mathrm{~mm}$ (b): Simulated scattering parameters of the filter. 


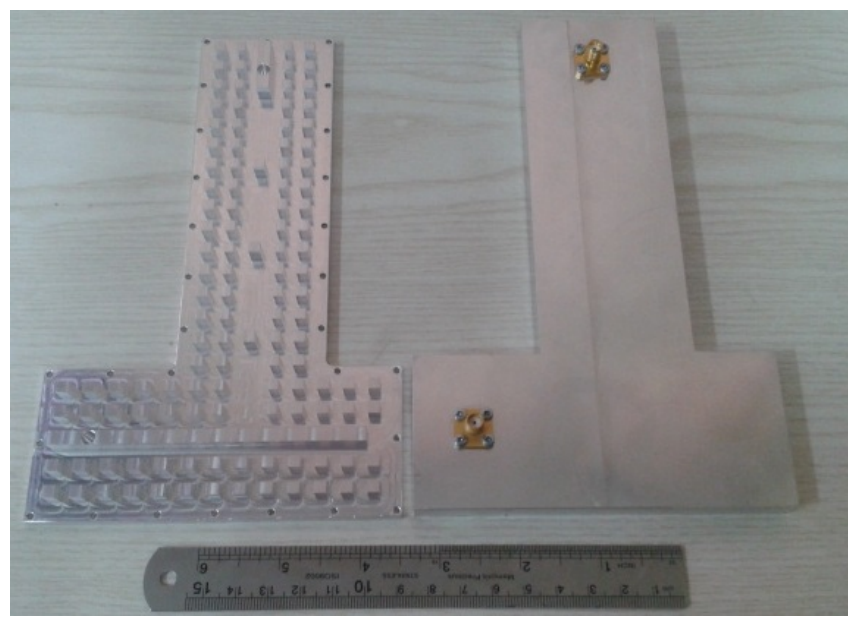

Fig. 8. (a): Fabricated fourth degree bandpass filter in center frequency of $11.59 \mathrm{GHz}$ and bandwidth of $72 \mathrm{GHz}$.

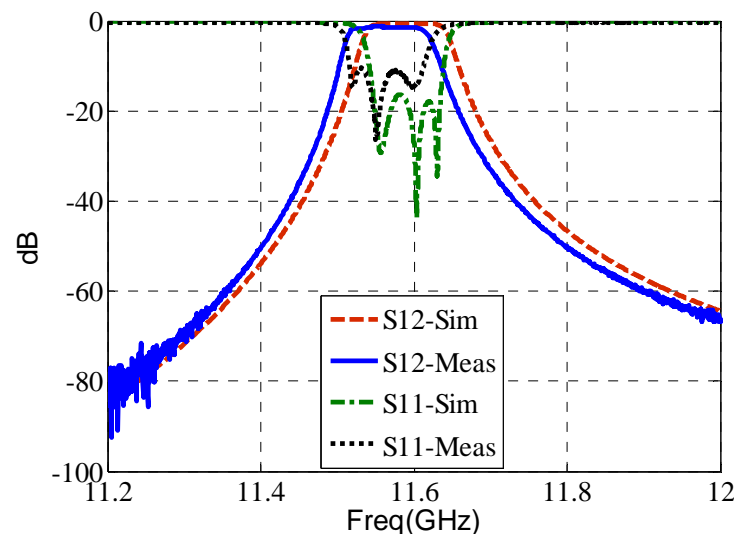

(a)

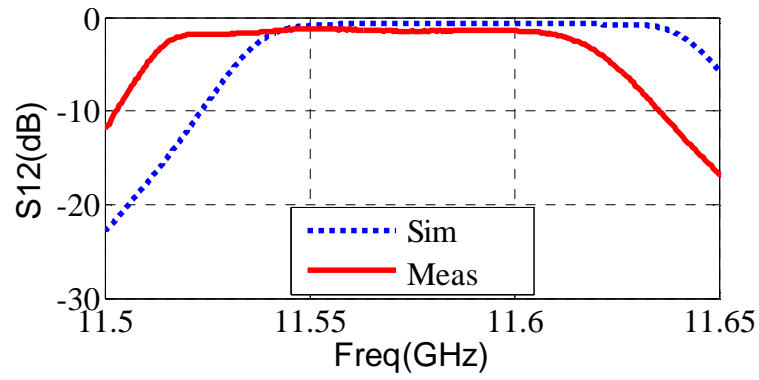

(b)

Fig. 9. (a): Measured and simulated $S_{11}$ and $S_{12}$ of the design. (b): Measured and simulated $S_{12}$ of the design in the passband.

presented in Fig. 9. Generally there is good agreement between the measurement and simulation. The decrease in the center frequency is caused by tolerance in fabrication. The measured insertion loss in the passband is higher about $0.7 \mathrm{~dB}$ in contrast with the simulation that is caused partially by the losses and mismatch of the SMA connectors. It has been shown previously [2] that designing and using a proper TRL calibration kit will result in lower insertion loss. Also, the roughness of the inner surface may cause the additional measured loss. Silver plating can eliminate this factor and also will increase the Q-factor of the cavities. Moreover, cavity sizes will be reduced, the decrease in the center frequency can be compensated.

\section{CONCLUSION}

This paper described a novel design of a filter on a ridge gap waveguide. The design concept was based on creating a defect in a periodic structure as in crystal photonics. The idea was applied to the ridge gap waveguide and a bandstop filter was realized. The procedure for the optimal design of the cavity was explained using modal analysis. A bandpass filter was designed by introducing the second ridge at the vicinity of the cavity. The coupling between the cavity and the ridge, which guided the wave to the filter's second port, was optimized. A sample model of a forth order filter with 72 $\mathrm{MHz}$ bandwidth at the center frequency of $11.59 \mathrm{GHz}$ was designed, manufactured and measured. The couplings among the cavities were adjusted by the height of the pins. The design work to develop a multi-channel filter is on the way.

\section{ACKNOWLEDGMENT}

This research work has been conducted at the Wireless Terminal Test Lab. K. N. Toosi University of Technology. The authors would like to acknowledge Iran National Science Foundation (INSF) for financial supports.

\section{REFERENCES}

[1] P-S. Kildal, E. Alfonso, A. Valero-Nogueira, E. Rajo-Iglesias, "Local metamaterial-based waveguides in gaps between parallel metal plates,” IEEE Antennas Wireless Propag. Lett., vol. 8, pp. 84 - 87, 2009.

[2] P-S. Kildal, A. U. Zaman, E. Rajo-Iglesias, E. Alfonso, A. ValeroNogueira, "Design and experimental verification of ridge gap waveguide in bed of nails for parallel-plate mode suppression,” IET Microwaves Antennas Propag, vol. 5, Iss. 3, pp. 262-270, Feb 2011.

[3] E. Rajo-Iglesias, P-S. Kildal, "Numerical studies of bandwidth of parallel plate cut-off realized by bed of nails, corrugations and mushroom-type EBG for use in gap wave-guides," IET Microwaves Antennas Propag, vol. 5, no. 3, pp. 282-289, March 2011

[4] E. Rajo-Iglesias, A. Uz Zaman, P-S. Kildal, "Parallel plate cavity mode suppression in microstrip circuit packages using a lid of nails," IEEE Microwave Wireless Components Lett., vol. 20, no. 1, pp. 31-33, Dec, 2009.

[5] A. Algaba Brazález, A. Uz Zaman, P.-S. Kildal, "Improved microstrip filters using PMC packaging by lid of nails", IEEE Transactions on Components, Packaging and Manufacturing Technology, vol. 2, no. 7, pp. 1075-1084, July 2012.

[6] S. J. Fiedziuszko, J. A. Curtis, S. C. Holme, R. S. Kwok, "Low loss multiplexers with planar dual mode HTS resonators,” IEEE Trans. Microw. Theory Tech., vol. 44, no. 7, pp. 1248-1257, July 1996.

[7] Y. Rong, H. Yao, K. Zaki, T. Dolan, "Millimeter-wave ka-band H-plane diplexers and multiplexers,” IEEE Trans. Microw. Theory Tech., vol. 47, pp. 2325-2330, Dec 1999.

[8] S. B. Cohn, “Direct coupled resonator filters," Proc. IRE, vol. 45, pp. 187-196, Feb 1957.

[9] L. Young, "Direct coupled cavity filters for narrow and wide bandwidths," IEEE Trans. Microw. Theory Tech., vol. 41, no. 5, pp. 162-178, May 1963.

[10] R. Levy, “Theory of direct coupled cavity filters,” IEEE Trans. Microw. Theory Tech., vol. 15, no. 6, pp. 340-348, Jun 1967.

[11] A. U. Zaman, A. Kishk, and P.-S. Kildal, "Narrow-band microwave filter using high Q groove gap waveguide resonators without sidewalls", IEEE Transactions on Components, Packaging and Manufacturing Technology, Vol. 2, No. 11, pp. 1882-1889, November 2012. 
[12] A.U. Zaman, T. Vukusic, M. Alexanderson, P.-S. Kildal, "Gap waveguide PMC packaging for improved isolation of circuit components in high frequency microwave modules", accepted for publication in IEEE Transactions on Components, Packaging and Manufacturing Technology, July 2013.

[13] A. U. Zaman, E. Rajo-Iglesias, E. Alfonso, P-S. Kildal, "Design of transition from coaxial line to ridge gap waveguide," Antennas and Propag. Society Int. Symp., 2009, pp. 1-4.

[14] D. W. Prather, S. Shi, A. Sharkawy, J. Murakowsky, G. J. Schneider, Photonic Crystals, New Jersey, WILEY 2009.

[15] D. England, I. Fushman, J. Vuckovic, "General recipe for designing photonic crystal cavities,” Optics Express, vol. 13, pp. 5961-5975, 2005.

[16] E. Pucci, A. U. Zaman, E. Rajo-Iglesias, P.-S. Kildal and A. Kishk, "Study of Q-factors of ridge and groove gap waveguide resonators", accepted for publication in IET Microwaves, Antennas \& Propagation, June 2013.

[17] E. Alfonso Alos, A. U. Zaman, and Per-Simon Kildal, "Ka-band gap waveguide coupled-resonator filter for radio link diplexer application", accepted for publication in IEEE Transactions on Components, Packaging and Manufacturing Technology, November 2012.

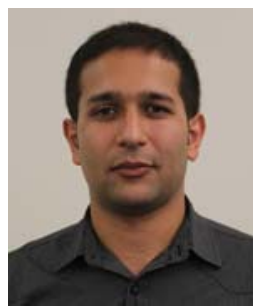

Milad Sharifi Sorkherizi was born in Tehran, Iran in 1988. He received his B.S. and M.S. degrees in Electrical Engineering from K. N. Toosi University of Technology, Tehran, in 2010 and 2012 respectively. He is now pursuing his studies toward Ph.D. degree in Concordia University, Montreal.

His research interests include filters, multiplexers, millimeter-wave guiding structures and leaky-wave antennas.

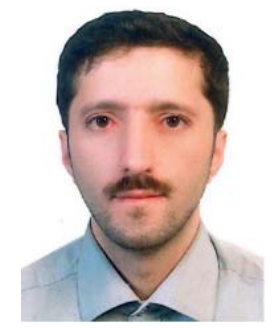

Ali Khaleghi Received his Ph.D. degree in physics from the University of Paris XI, France in 2006. He joined the Institut d'Electronique et de Télécommunications de Rennes (IETR), France, as a postdoctoral researcher working on time reversal wireless communications. $\mathrm{He}$ started a position at the Intervention Centre, Oslo University Hospital, Norway, in 2008. There, he worked on wave propagation inside and on the human body. He hold 60 publications including 18 scientific journals and two patents. Currently, he is an assistant professor at the K. N. Toosi University of Technology, Tehran, Iran. He is heading the wireless terminal test laboratory.
Per-Simon Kildal (M'76-SM'81-F'95) is professor in antennas at Chalmers University of Technology in Gothenburg, Sweden since 1989. He is heading the Antenna group. His main tasks are to lead and supervise research within antenna systems. Until now, 19 persons have received a Ph.D. from him. Kildal received himself two doctoral degrees from the Norwegian Institute of Technology in Trondheim.

Kildal has authored more than 120 articles in scientific journals; concerning antenna theory, analysis, design and measurements. Two articles were awarded best paper awards by IEEE (1985 R.W.P. King Award and 1991 Schelkunoff Prize Paper Award). In 2011 he received the prestigious Distinguished Achievements Award from the IEEE Antennas and Propagation Society.

Kildal has done the electrical design of the $40 \mathrm{~m} \times 120 \mathrm{~m}$ cylindrical reflector antenna and line feed of the EISCAT scientific organization, and the dual-reflector Gregorian feed of the $300 \mathrm{~m} \varnothing$ radio telescope in Arecibo. He is the inventor behind technologies such as dipole with beam forming ring, the hat antenna, and the eleven feed. All these feeds have been used in industry, and until now more than 930000 hat-fed reflectors have been manufactured for use in radio links. Kildal was the first to introduce the reverberation chamber as an accurate measurement instrument for Over-The-Air (OTA) characterization of small antennas and wireless terminals for use in multipath environments with fading. Kildal is also the originator of the concept of soft and hard surfaces from 1988, today being regarded as the first metamaterials concept. This concept is the basis of his newest and most fundamental invention, the gap waveguide technology. His research is innovative and industrially oriented, and has resulted in several patents and related spinoff companies, the most known being Bluetest AB.

Kildal organizes and lectures in courses within the European School of Antenna (ESoA, www.antennasvce.org). His textbook Foundations of Antennas - A Unified Approach (Lund, Sweden: Studentlitteratur, 2000) was well received, and is now in the process of being revised.

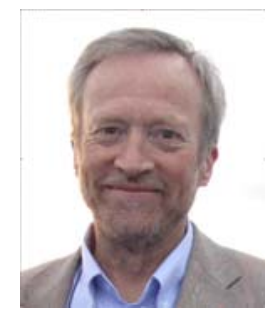

